\title{
Rerista Pueblos y

\section{INTERCAMBIO DE PLANTAS ENTRE HUERTOS Y OTROS ESPACIOS: ¿UNA ESTRATEGIA DE CONSERVACIÓN PARA EL BOSQUE MESÓFILO DE MONTAÑA DEL VOLCÁN TACANÁ, CHIAPAS, MÉXICO?}

María Eugenia Escobar Hernández Estudiante de doctorado del ECOSUR - San Cristóbal mescobar@ecosur.edu.mx

Eduardo Bello Baltazar ECOsur - San Cristóbal ebello@ecosur.mx

Erin Ingrid Jane Estrada Lugo ECOSUR - San Cristóbal eestrada@ecosur.mx

\section{RESUMEN}

Se analiza el papel que realiza una de las prácticas de conservación en los huertos de una comunidad mame en la Reserva de Biosfera Volcán Tacaná; Chiquihuite, Unión Juárez, Chiapas. Lo anterior, se llevó a cabo mediante la recolecta e identificación de las especies presentes en veinticuatro huertos de la comunidad y entrevistas semi estructuradas sobre el espacio de origen y el uso a la especie. Con esta información se elaboró una matriz de presencia-ausencia y una red de relaciones entre espacios, y se concluyó que existe una práctica de intercambio, principalmente entre los espacios huerto-montaña y huerto-huerto. La base de este intercambio es el uso y por ello la definición local del concepto de conservación, no se explica sin este concepto.

PALABRAS CLAVE: uso de plantas, redes, intercambio, reserva de la biosfera.

\section{INTERCHANGE OF PLANTS BETWEEN HOME GARDENS AND OTHER SPACES: A CONSERVATION STRATEGY FOR THE CLOUD FOREST OF THE TACANÁ VOLCANO IN CHIAPAS STATE, MEXICO?}

\section{ABSTRACT}

This paper analyzes the role played by one of the conservation practices carried out in the home gardens of a Mame community in the Tacaná Volcano Biosphere Reserve: Chiquihuite, in the Unión Juárez municipality of Chiapas State. This analysis was conducted through collecting and identifying the species present in 24 of the home gardens of the community as well as semi-structured interviews regarding the space of origin and use of the species. Based on this information, a presence-absence matrix was developed as well as a network of relationships between spaces. It was concluded that there is a practice of interchange, mainly between home garden and mountain, and between home garden and home garden. This interchange is based on use and therefore the local definition of conservation cannot be explained without this concept.

KEY WORDS: plant uses, networks, interchange, biosphere reserve. 


\section{INTRODUCCIÓN}

E

xisten distintos significados de la conservación. El concepto ha sido abordado por profesionales de la biología y de la ecología, principalmente. En este sentido, una de las perspectivas indica que desde la década de los años ochenta, la biología de la conservación ha considerado que los disturbios humanos han afectado la biodiversidad en las poblaciones y comunidades biológicas (Soulé 1985). De allí que la definición más usada sobre este tema nos indique entender la conservación como «la forma de manejo de la biosfera de tal manera que se procure el máximo beneficio para las generaciones actuales, pero que a la vez se mantenga el potencial para satisfacer las necesidades de las futuras generaciones» (WRI 1992:285). Sin embargo, este enunciado no aclara que exista una manera específica o exclusiva para acceder al manejo de la biosfera.

En México, se considera qué las Áreas Naturales Protegidas (ANP) son uno de los instrumentos diseñados para armonizar el enfoque de conservación con el desarrollo local y regional (Halffter 1992). Una de sus categorías, las Reservas de Biosfera (RB), ha sido calificada como un espacio que pretende resolver los conflictos de intereses entre la población y las iniciativas de conservación. También considera al ser humano como parte de los ecosistemas (Halffter 1992). No obstante, en la práctica, la administración de las RB continúa asumiendo las prácticas humanas como principales responsables del deterioro ambiental. Algunas de estas actividades están relacionadas con el uso de los recursos naturales (Lazos y Paré 2000, Durand 2003, Paz 2005).

Los huertos son espacios en que es posible ver representado el uso constante del ambiente (Galluzi et al. 2010). Se trata de lugares cercanos a la vivienda en donde las personas cultivan una gran diversidad de plantas para satisfacer sus necesidades diarias de consumo (Barrera 1981); asimismo, representan sistemas diversificados y sustentables que integran una interacción cercana entre la naturaleza y la cultura expresada a través del uso. Con todo, también son espacios mayoritariamente relacionados como reservorios de agrobiodiversidad y ejemplo de los sistemas alimentarios de las comunidades. Poco han sido entendidos y utilizados como áreas estratégicas de conservación (Galluzi et al. 2010).

En el presente artículo se aportan datos de investigación de una práctica de intercambio que promueve el flujo de plantas en espacios con vegetación característica de bosque mesófilo de montaña (BMM) y aquellos donde es evidente el uso de la biodiversidad, tales como el huerto. El intercambio, hoy en día, se reconoce 
cada vez más como una estrategia de dispersión y conservación vegetal (Ellen y Platten 2011). Lo anterior es significativo para el BMM, ya que las especies que lo conforman son muy sensibles a la perturbación y no se recuperan fácilmente (Ramírez-Marcial et al. 2010). En México, estos bosques abarcan una superficie menor a $1 \%$ del territorio nacional, y en Chiapas solamente se distribuyen en pequeños fragmentos de la Sierra Madre, las Montañas de Oriente y las Montañas del Norte (Rzedowski 1983, Ramírez-Marcial et al. 2010, González-Espinosa et al. 2011). En la comunidad de estudio, Chiquihuite (Unión Juárez), que se ubica dentro de la Reserva de la Biosfera Volcán Tacaná (6378 hectáreas), el BMM ocupa $27.69 \%$ de la superficie total. Hay 33 hectáreas de BMM en buen estado de conservación y 122 con vegetación secundaria.

Por datos como los anteriores, se reconoce al BMM como el tipo de vegetación con el área más reducida de todos los ecosistemas presentes en México (CONANP 2008). El objetivo de este trabajo es analizar cómo interviene el intercambio de plantas entre huertos y otros espacios como estrategia de conservación de especies vegetales presentes en el bosque mesófilo de montaña. Al parecer, las redes de intercambio entre espacios pueden funcionar cuando, por el uso, las especies se mueven de su sitio original. Se partió de la hipótesis de que los huertos de la comunidad de Chiquihuite son espacios abiertos, que interaccionan con otros, es decir, que están lejos de constituir espacios aislados. En la base de tales interacciones está la acción del intercambio de plantas en la vida cotidiana de la población, la cual es relevante para la conservación.

\section{METODOLOGÍA ZONA DE ESTUDIO}

Chiquihuite se ubica a $15^{\circ} 05^{\prime} 40^{\prime \prime}$ de latitud norte, $92^{\circ} 06^{\prime} 01^{\prime \prime}$ de longitud oeste, y a 2080 metros sobre el nivel del mar (msnm), en uno de los puntos más altos de la Sierra Madre de Chiapas, Municipio de Unión Juárez, cerca del Volcán Tacaná (Escobar 2009). Es una localidad de 597.38 hectáreas (figura 1). Se compone de pequeñas propiedades privadas y dentro de ella existen 54 casas (CONANP 2008). Su población es de 392 habitantes, de los cuales 205 son mujeres y 187, hombres (IMSS 2012). Se consideran hablantes de la lengua mam (IMSS 2012). La principal actividad económica es la floricultura y la agricultura de autoabasto de productos como maíz, chilacayote y papa (Escobar 2009, Cruz 2010). 


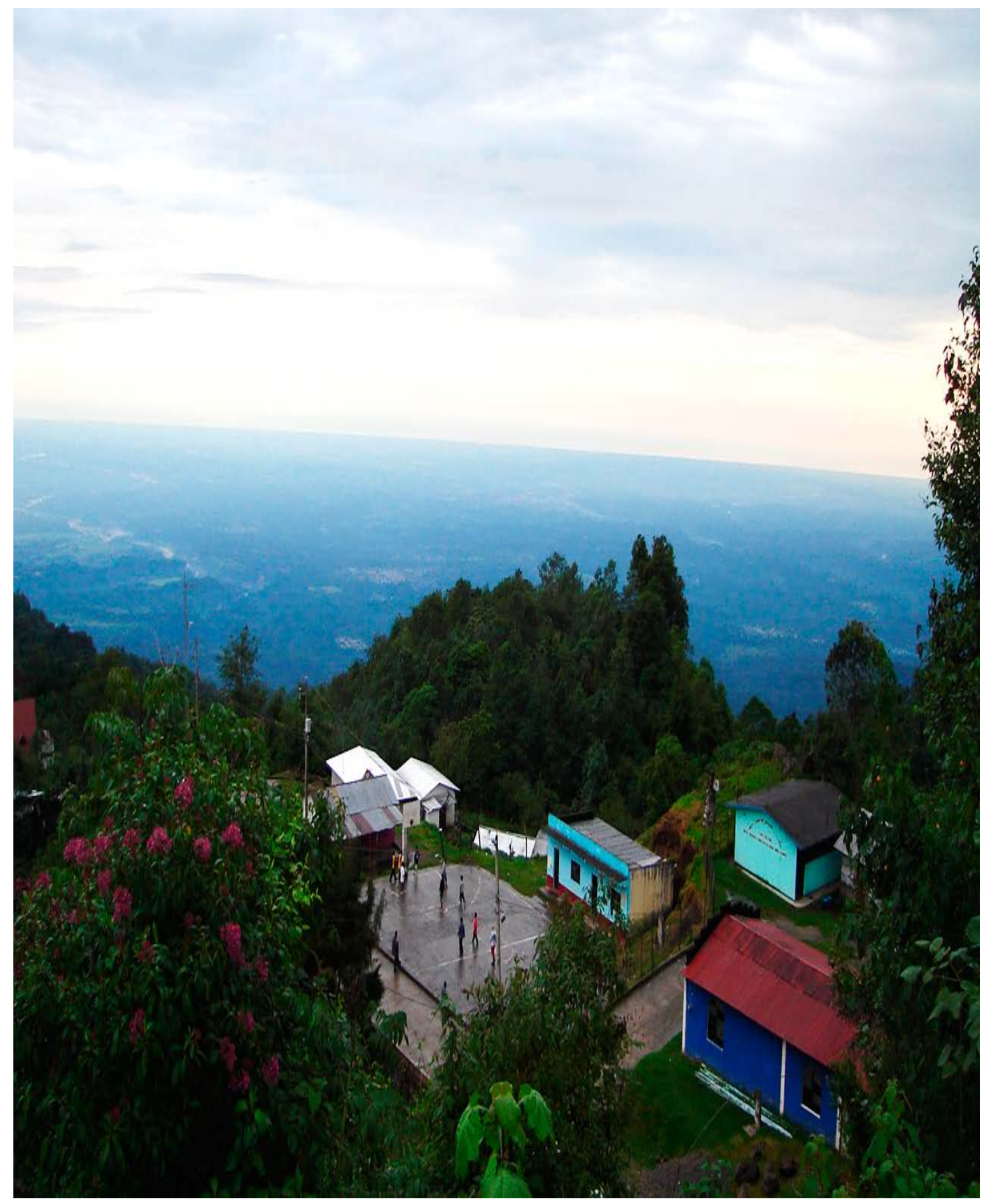

Figura 1. Zona de servicios de la comunidad de Chiquihuite, Unión Juárez, Chiapas. Foto: Eugenia Escobar. 
El clima es templado húmedo con abundantes lluvias en verano, con una temperatura media de $15.3^{\circ} \mathrm{C}$ y alrededor de $1473 \mathrm{~mm}$ de precipitación anual. El tipo de suelo predominante es el andosol, el cual se ha formado a partir de cenizas volcánicas, y es muy susceptible a la erosión (CONANP 2008).

\section{RECOPILACIÓN DE DATOS}

El trabajo de campo se realizó durante agosto de 2011 y desde febrero hasta mayo de 2012. Los huertos se seleccionaron de acuerdo con el tipo de informante que podría resultar propietario de estos espacios. Así, se dividieron entre informantes clave (14 entrevistados) y de calidad (10 entrevistados). Según Hammersley y Atkinson (1994), los informantes clave son aquellas personas que tienen disponibilidad de tiempo y facilidad para aportar información. Y los informantes de calidad son aquellos individuos que pueden aportar información eficiente y de primera mano sobre la temática principal: el uso y origen de las plantas en el huerto.

El tamaño de muestra $(n=24)$ se determinó como tal debido a que representa casi $50 \%$ del total de huertos en la comunidad. Lo anterior tuvo como finalidad obtener una muestra que representara la variación local de especies que existen dentro de este espacio. Los huertos seleccionados están distribuidos en toda el área de la comunidad (figura 2).

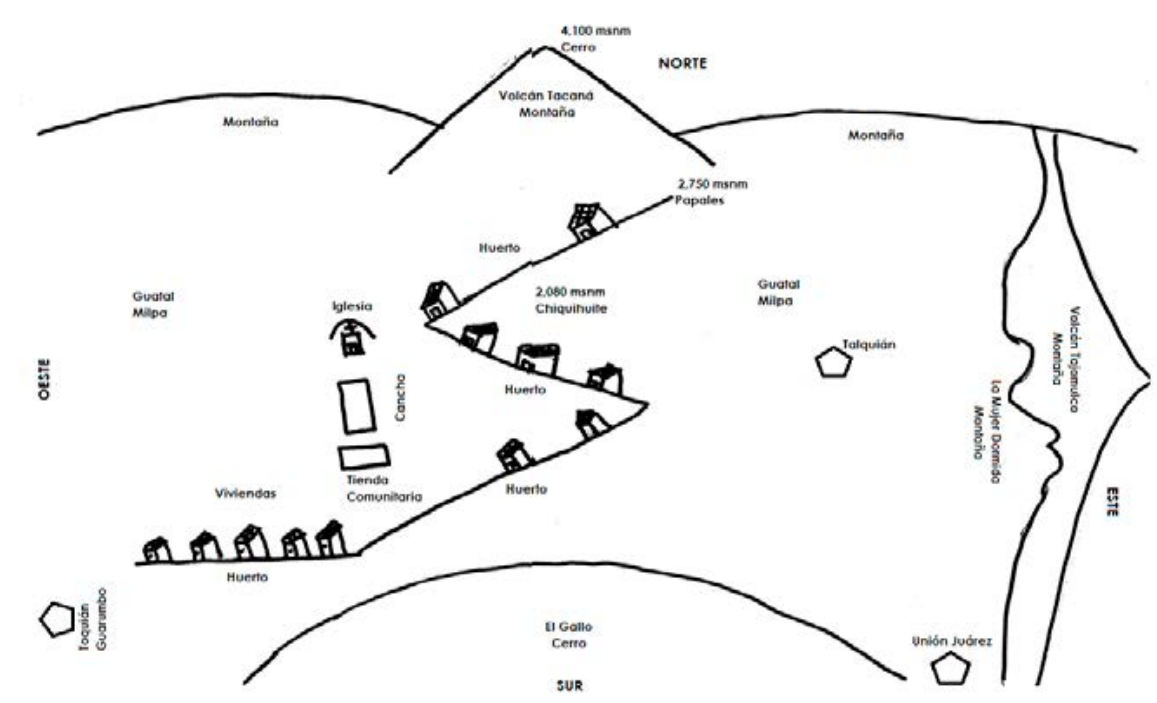

Figura 2. Ubicación de los espacios de uso de los recursos naturales en la comunidad de Chiquihuite, Unión Juárez, Chiapas. Fuente: elaboración propia. 
El espacio es el objeto sobre el cual la población manifiesta sus intenciones sobre el ambiente. Es decir, es la materia prima que antecede a todo conocimiento y a toda práctica (Raffestin 1980). De acuerdo con el conocimiento local de la comunidad de Chiquihuite (Escobar 2009), los espacios que se pueden encontrar son: 1) la montaña, lugar en donde existen más árboles (estrato arbóreo) y hay en menor proporción «cascajo» (estrato arbustivo); 2) el cerro, compuesto principalmente por cascajo y hierbas; 3) El «guatal», en otros lados también llamado acahual o tierras de descanso, es el espacio que deja de ser utilizado después de la cosecha de la milpa; 4) el huerto, sitio, jardín o «alhuerte», destinado para el cultivo de flores, hortalizas, plantas medicinales y plantas comestibles; 5) la milpa, donde se siembran los principales productos de autoconsumo (figura 3).

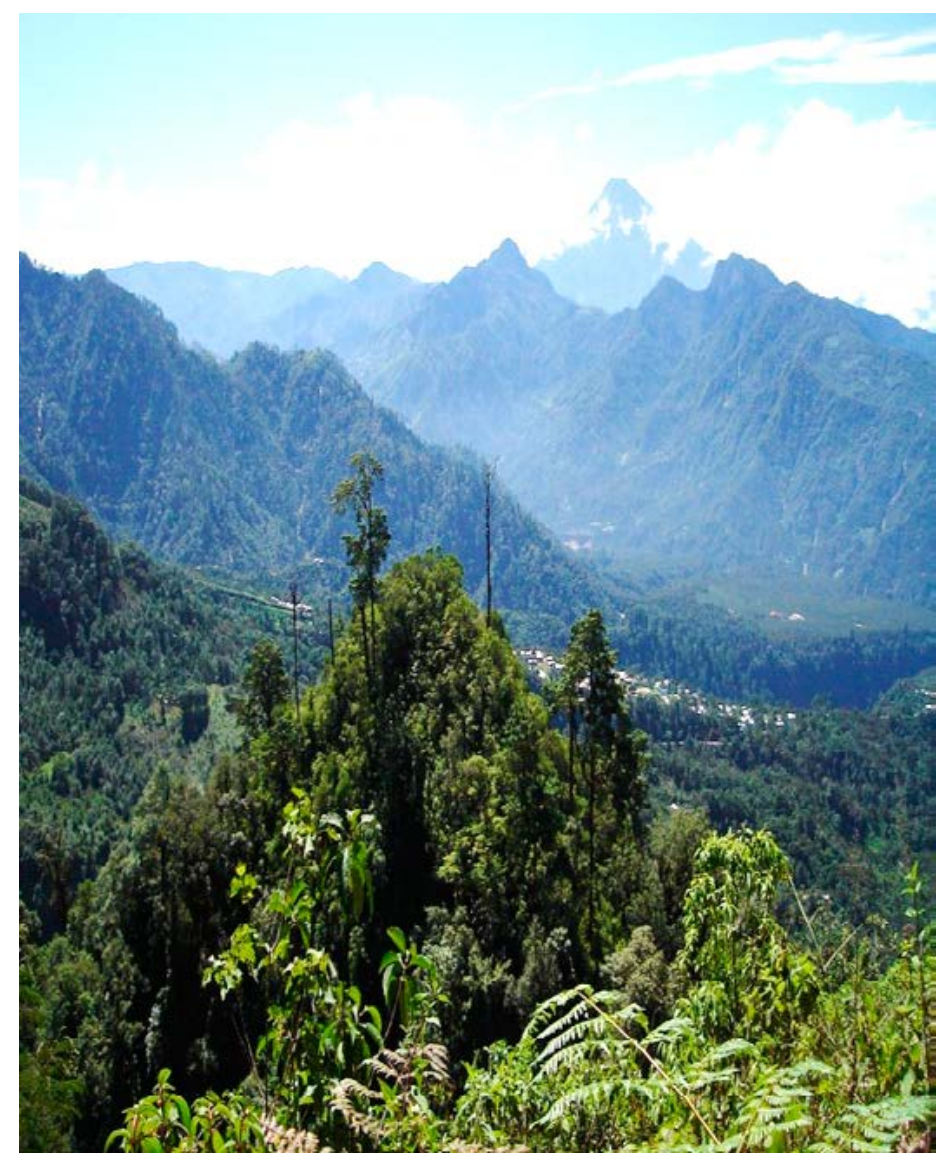

Figura 3. Perfil de la comunidad de Chiquihuite en donde pueden apreciarse los espacios cerro y montaña. Al frente se observa el Cerro del Gallo, detrás se nota la comunidad de Talquian y al fondo sobresale la montaña "Mujer dormida" y el Volcán Tajomulco de Guatemala. Foto: Eugenia Escobar. 
Además de los espacios proporcionados por Escobar (2009), por los datos que aportaron los informantes consultados se agregaron los tres siguientes: los viveros gubernamentales, otra comunidad y el espacio local. Del primero se entiende que las especies encontradas se obtuvieron de algún programa de gobierno. Del segundo se registró que la especie es comprada o intercambiada en comunidades de Chiapas (Cacahoatán, Unión Juárez y San Cristóbal de las Casas) o de Guatemala. Del tercero se reportó que la especie nace en el huerto por medio de la dispersión por viento, agua y/o animales silvestres.

Con esta información de base se llevaron a cabo recorridos y durante ellos se aplicaron entrevistas semiestructuradas cuyas preguntas temáticas se centraban en conocer los espacios de los que se han obtenido las especies vegetales que están en el huerto y el uso que se les brinda. En el día a día se estableció un mayor acercamiento para estos dos tópicos por medio de la observación participante (Taylor y Bogdan 1996). Todos estos datos fueron registrados en un diario de campo y solamente se realizaron grabaciones digitales si la persona entrevistada lo autorizaba. Ambos registros se transcribieron fielmente (Ruiz e Izpizúa 1989).

Se hicieron recolectas de las plantas que eran reconocidas por los entrevistados. Los materiales fueron procesados e identificados en el Herbario CHIP del Instituto de Historia Natural y Ecología (IHNyE). Asimismo, se determinó si las especies eran nativas, neotropicales e introducidas. En las primeras se consideró las especies de la flora natural del sur de México. En las segundas, las provenientes del trópico americano, exceptuando las del sur de México, y en las terceras, las especies que provienen del norte del continente americano y los demás continentes (lbarra 1996).

Se cotejaron los nombres científicos con el apoyo de la aplicación Taxonstand, utilizada para reconocer la correcta escritura de taxones de plantas en el programa R versión 2.15.1 (R Development Core Team 2014). De la misma manera, la información fue comparada especie por especie con la base de datos de Tropi$\cos \circledast$ (tropicos.org).

Las formas de crecimiento se agruparon, según Ghazoul y Sheil (2010), en árboles, arbustos, herbáceas, epífitas, rastreras y trepadoras. Fueron definidas como árboles aquellas especies con tallo leñoso mayores a 5 metros de altura. Los arbustos, como plantas de baja estatura (menores a 5 metros), usualmente con tallo leñoso. Las herbáceas, como los organismos vegetales que no poseen tallo leñoso y son usualmente terrestres. Las epífitas, como aquellas plantas con tallo no leñoso que crecen sobre otras plantas vivas. Las rastreras, como plantas 
que extienden sus raíces sobre el suelo. Las trepadoras son las que utilizan sus raíces para sostenerse de su huésped y las parásitas son las que dependen de los nutrientes orgánicos que les aporta el hospedero.

Los datos recopilados de los informantes se agruparon en siete categorías que hacen alusión al uso de las plantas según el sistema propuesto por Cook (1995): 1) Combustible: plantas usadas como leña y carbón. 2) Comestible: incluye las bebidas. 3) Materiales: para construcción, herramientas, cercos, utensilios, artesanías y otros usos domésticos. 4) Medicinal y veterinaria. 5) Tóxica. 6) Uso ambiental: ornamental, sombra, control de erosión y humedad. 7) Uso social: religioso, ritual o ceremonial.

Con base en la Convención Internacional para el Comercio de Especies Amenazadas 2010 (CITES, por sus siglas en inglés), en la Norma Oficial Mexicana NOM059-2010 y a la Lista Roja 2012 de la Unión Internacional para la Conservación de la Naturaleza, IUCN por sus siglas en inglés; se determinó si las especies pertenecían a alguna categoría de atención a la conservación (CITES 2010, SEMARNAT 2010, UICN 2012).

\section{ANÁLISIS DE DATOS}

De la información obtenida se elaboró en primera instancia una matriz de presencia-ausencia de especies vegetales en una hoja de cálculo. Posteriormente, con los datos sobre el espacio de procedencia y con el número de interacciones de cada huerto con cada espacio, el intercambio pudo ser analizado por medio del análisis de redes (Ellen y Platten 2011). Por esta razón, se elaboró una representación gráfica de una red con el programa Cytoscape 2.8.2 (Shannon et al. 2003).

Para tal efecto, se representan dos tipos de nodos: los de origen y los de destino. Los primeros se refieren a los espacios de uso de los cuales se obtuvieron las especies. Los segundos representan los 24 huertos muestreados (estos se identifican por una letra que va de la «A» a la «X»). Ambos nodos están unidos por un puente que indica una interacción, que significa la frecuencia de la especie vegetal obtenida de cada espacio de uso. Estas mismas conexiones indican un movimiento unidireccional de los diferentes espacios de uso hacia los huertos muestreados.

En relación con los datos de riqueza de especies, estos se analizaron cuantitativamente por medio de las relaciones numéricas de la matriz de presencia-ausen- 
cia y se expresaron en términos de porcentajes. El análisis del material discursivo obtenido de las entrevistas y la observación participante se realizó a través de la categorización de dicha información.

\section{PRINCIPALES HALLAZGOS \\ RIQUEZA LOCAL}

Se registraron 109 especies, de las cuales 37 están presentes en los BMM. De estas se identificó $77 \%$ a nivel de especie. Se encontraron distribuidas en 49 familias. Las familias con mayor número de especies fueron: Orchidaceae (11), Asteraceae y Solanaceae (10), Bromeliaceae (7) y Rosaceae (6).

Las especies encontradas pueden ser categorizadas, en primera instancia, de acuerdo con su origen. Se representan en número, de mayor a menor, en nativas, neotropicales e introducidas. Para las especies no identificadas no fue posible su determinación taxonómica debido a que no se colectaron sus estructuras reproductivas. Por lo anterior, no se lograron incluir en esta gráfica (figura 4).

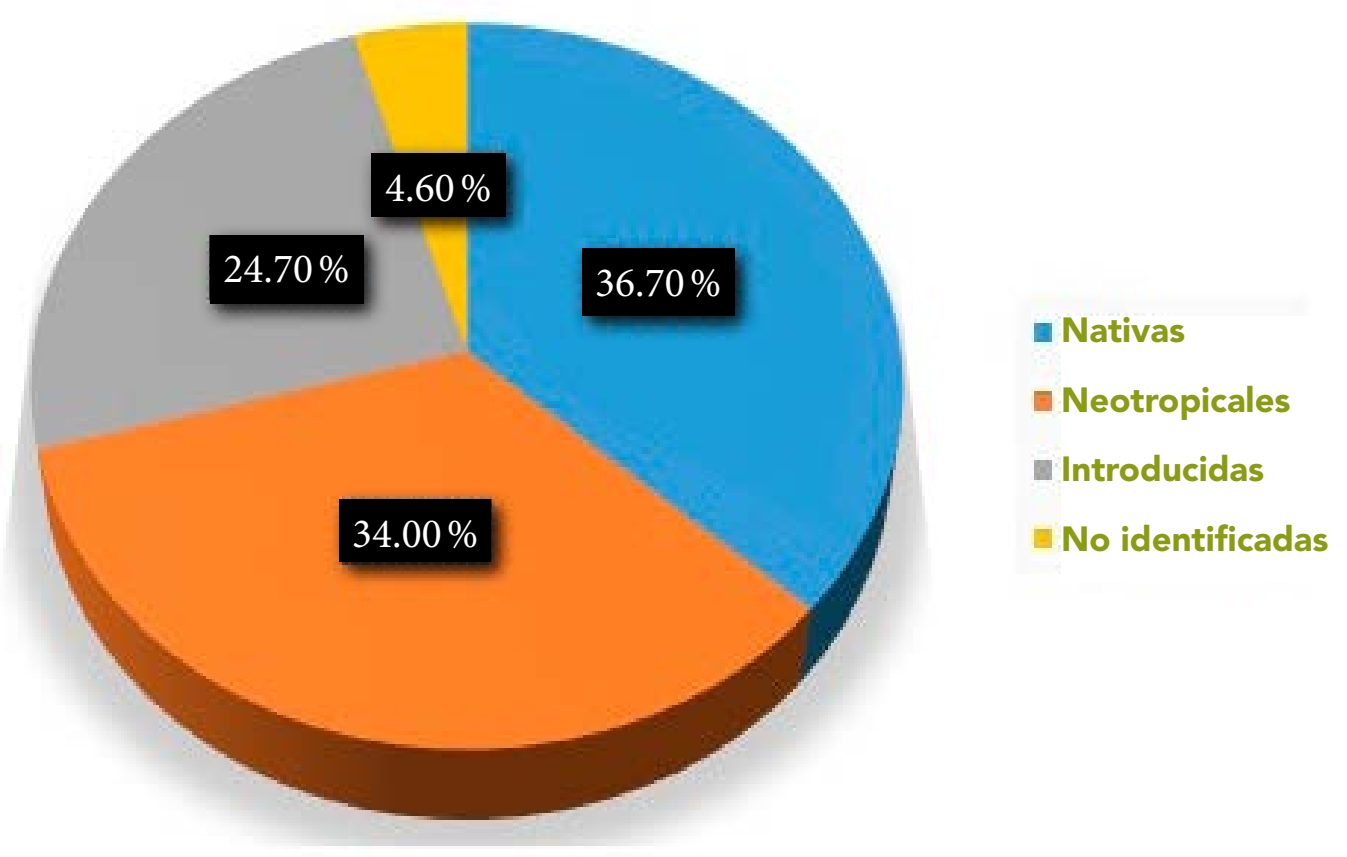

Figura 4. Porcentaje de especies registradas por categoría de origen en los huertos de la comunidad de Chiquihuite, Unión Juárez, Chiapas. 
De las formas de crecimiento, se encontró que las plantas herbáceas, arbóreas, epífitas y arbustivas son predominantes en los huertos de la comunidad, mientras que las rastreras, trepadoras y parásitas se encuentran representadas en menor porcentaje (figura 5).

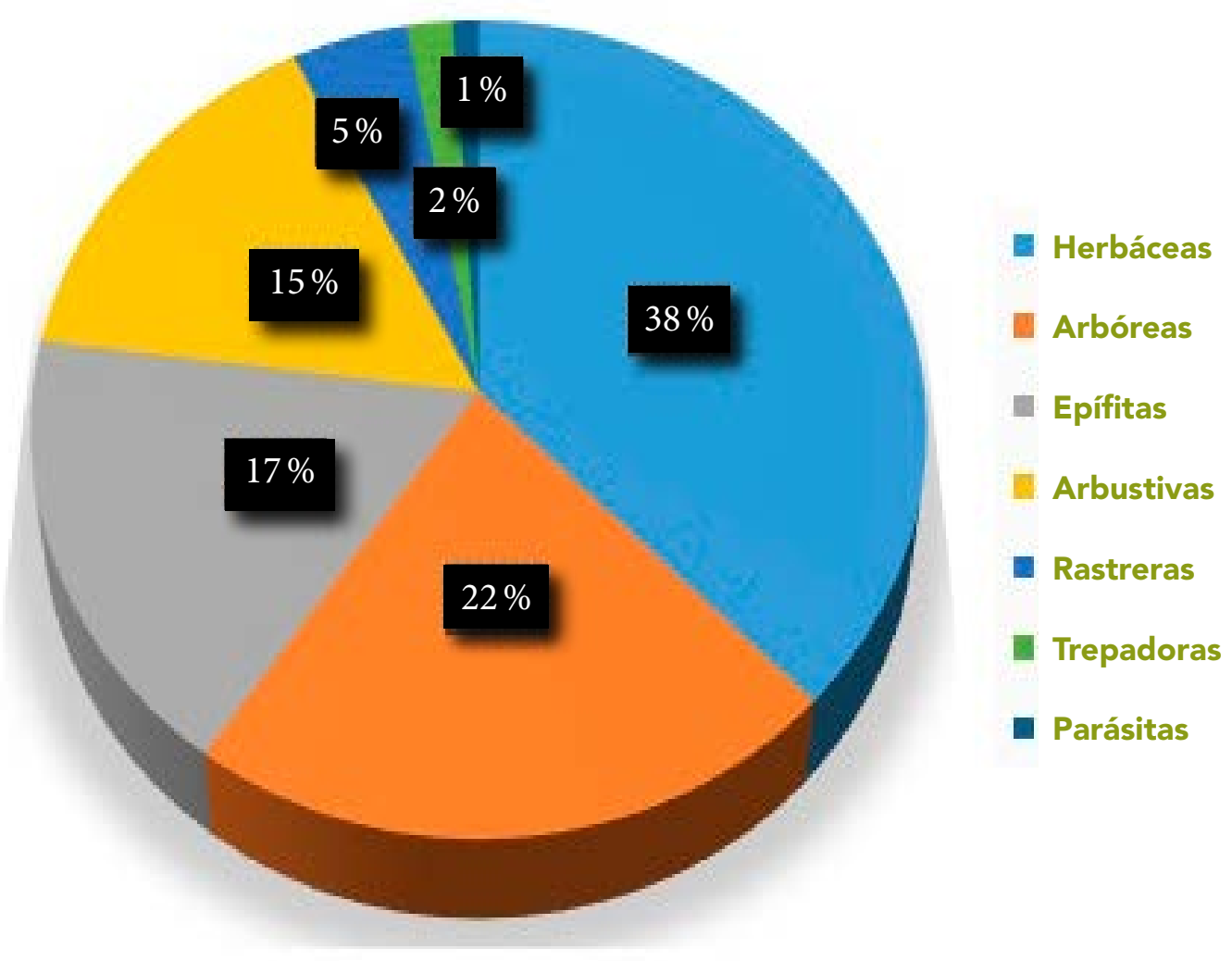

Figura 5. Porcentaje de especies por forma de crecimiento en los huertos de la comunidad de Chiquihuite, Unión Juárez, Chiapas.

Los usos recopilados se encuentran en mayor porcentaje en ambiental y comestible. Las plantas con uso social tienen menor presencia en los huertos. En la categoría plantas tóxicas no se registró ningún individuo (figura 6).

En cuanto al estatus de conservación, casi 30\% de las 109 especies registradas se encuentran categorizadas bajo la Lista Roja de la UICN, el CITES o la Norma Oficial Mexicana NOM-059-2010. 


\section{RED DE RELACIONES ENTRE ESPACIOS}

Las especies interaccionan dentro de los siete espacios de uso encontrados. Se reconoció un total de 409 interacciones, las cuales son: el huerto, 130 interacciones; montaña, 98; otra comunidad, 73; espacio local, 48; cerro, 31; viveros gubernamentales, 21; y milpa, 8 (figura 7). En el caso de los huertos $G, H, K, L, O, P, Q$, $R, V$ y $W$ tienen mayor interacción con el espacio huerto. Los huertos $A, B, C, D$, $E, I, S, U$ y $X$ presentan una mayor interacción con la montaña. Los huertos J, F y $\mathrm{N}$ tienen el mismo porcentaje de interacción entre la montaña y el huerto. Solamente el huerto $\mathrm{M}$ presentó una mayor interacción con el espacio «otra comunidad» (figura 8). Con esto, podemos afirmar que en los huertos de la comunidad de Chiquihuite se establecen interacciones de distinto grado con los espacios. Aunque a nivel de interacciones la relación entre huertos es superior, el número de especies provenientes de la montaña sigue siendo mayor que en el resto de los espacios de uso (45 especies), y de estas, 37 son características del BMM (figura 9). Se observa que de manera general existe una mayor conexión entre las relaciones huerto-huerto y huerto-montaña. El espacio - huerto- presenta comparativamente poca interacción con el resto de los espacios de uso.

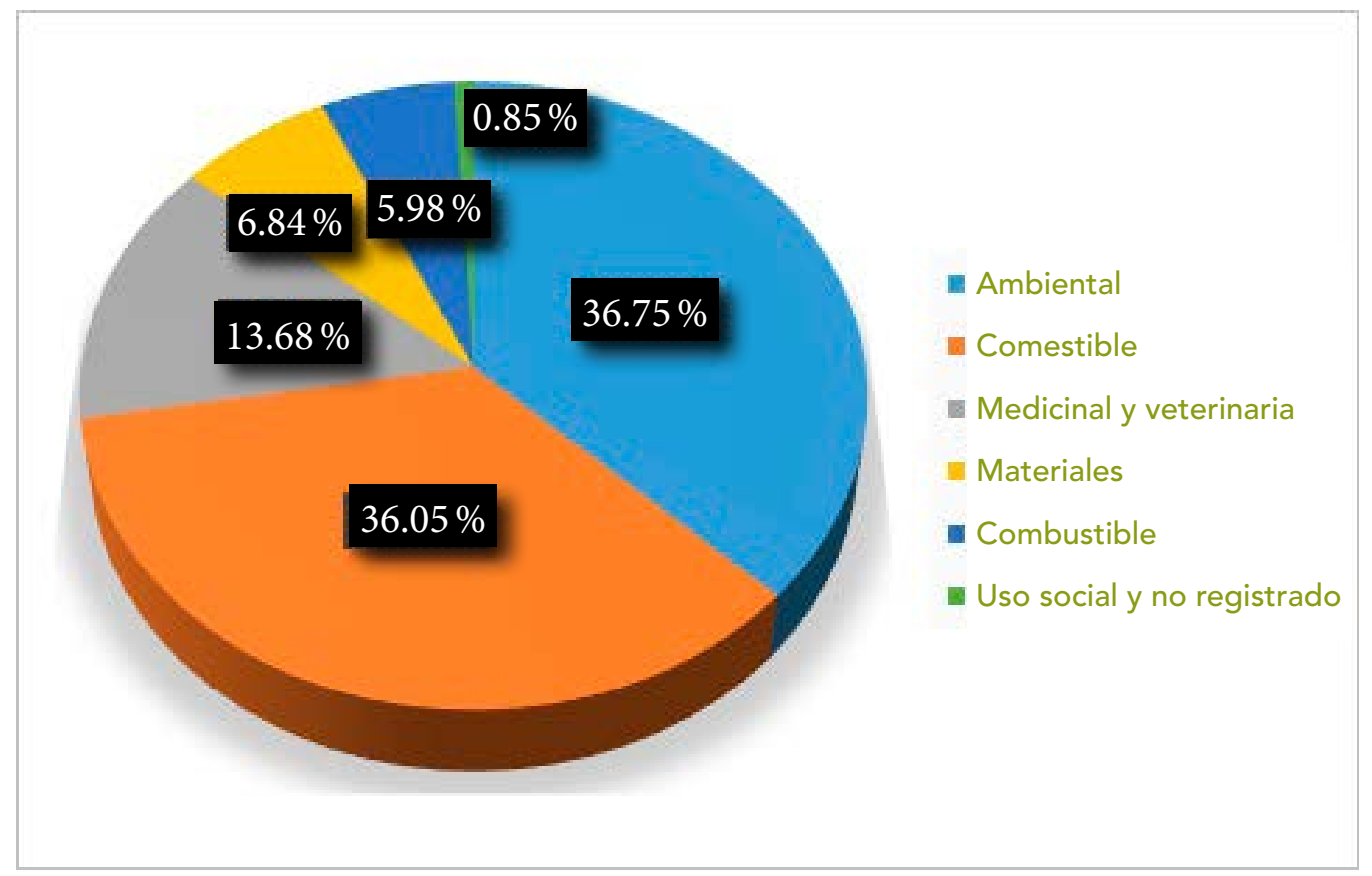

Figura 6. Principales usos de las plantas presentes en los huertos de la comunidad de Chiquihuite, Unión Juárez, Chiapas. 
La conexión entre huertos puede ocurrir de entre siete hasta 41 especies por huerto. Asimismo, cada especie puede interaccionar hasta 24 veces. Las que se comportan así con mayor frecuencia lo hacen desde 10 hasta 20 veces (cuadro 1). De estos taxones, Alnus acuminata, Saurauia aspera, Baccharis vaccinioides y Tillandsia guatemalensis son representativos del BMM (Breedlove 1981, Ramírez-Marcial et al. 2010, González-Espinosa et al. 2011).

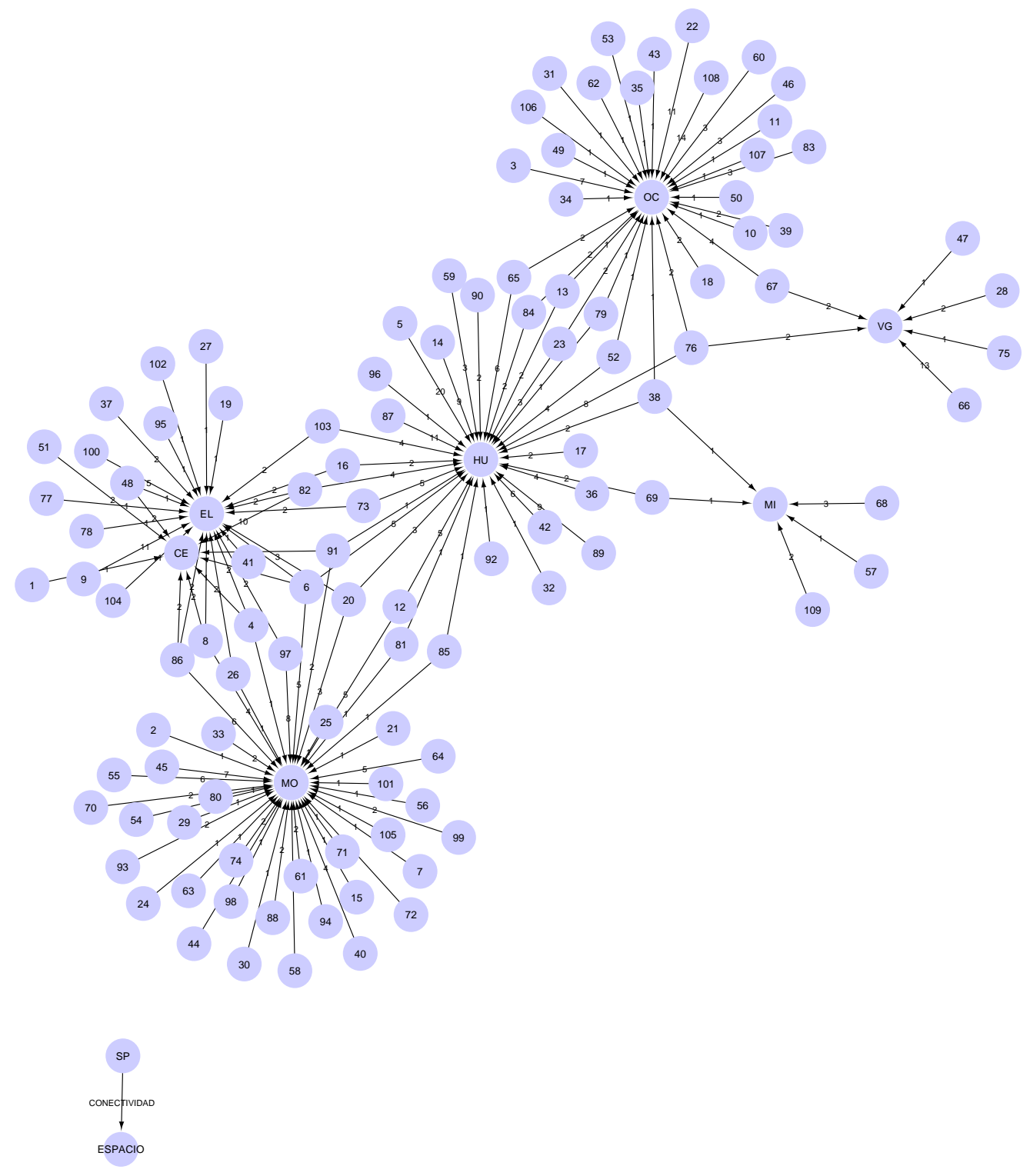

Figura 7. Conectividad de las especies presentes en los huertos de la comunidad de Chiquihuite, Unión Juárez, Chiapas. 


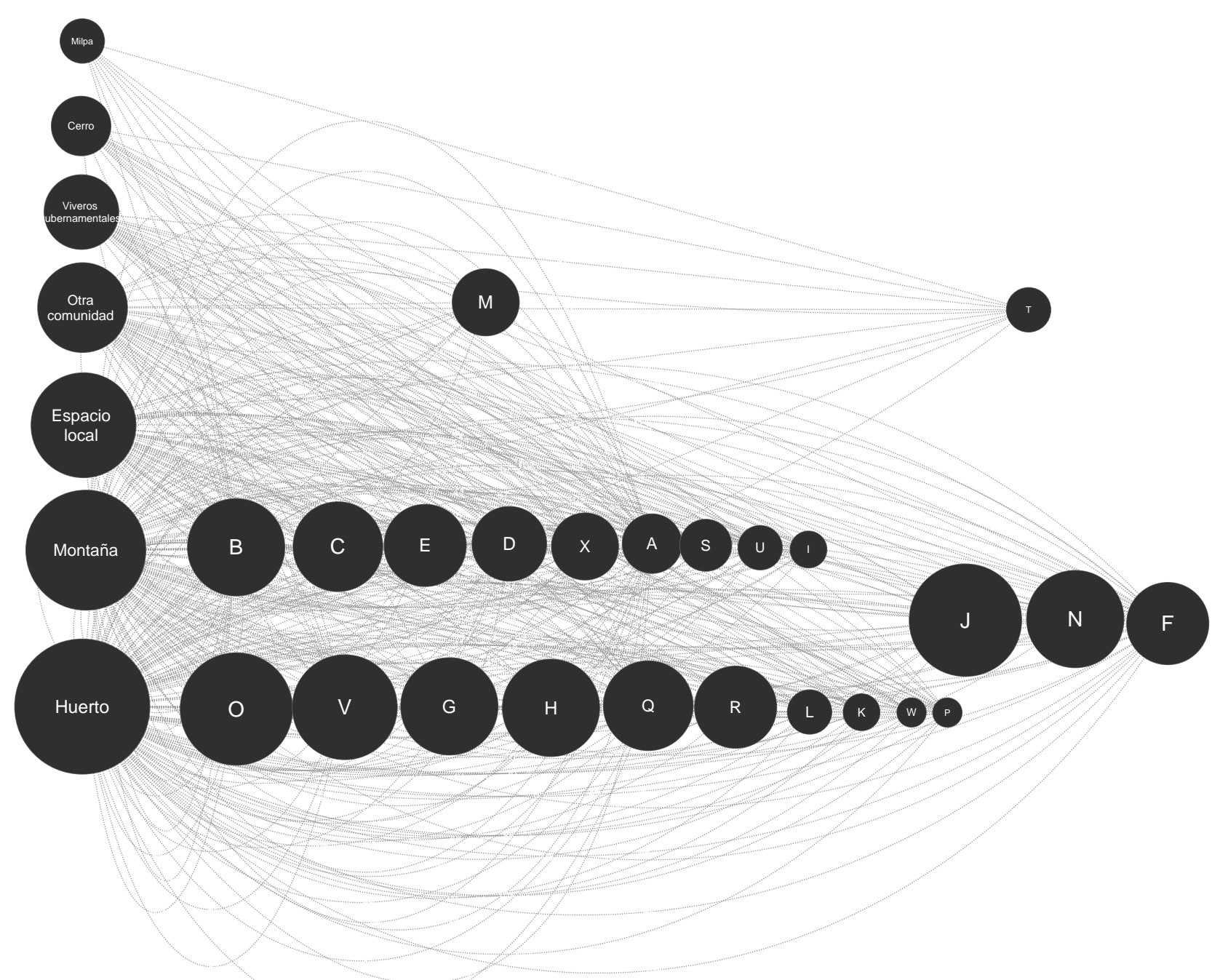

Figura 8. Conectividad entre huertos y espacios de uso de la comunidad de Chiquihuite, Unión Juárez, Chiapas.

\section{EL HUERTO DENTRO DE LA RED}

El reconocimiento de la riqueza y procedencia de especies indicó que el huerto no es un espacio cerrado, es un lugar que interacciona con otros espacios, principalmente, huerto-huerto y huerto-montaña. Además, contiene especies típicas del BMM. 
El análisis de redes indica un proceso de intercambio representado en la interacción entre los huertos y el resto de los espacios. Estas interacciones responden a un atributo de las redes que se conoce como conectividad, la cual se define como el número de puentes o enlaces que conectan un nodo focal con otros tipos de nodos (Proulx et al. 2005). En la figura 8, el número de enlaces que conectan al huerto con el huerto y al huerto con la montaña son mayormente visibles. Por lo tanto, consideramos que estos dos espacios poseen un grado de conectividad superior. Sin embargo, el número de especies presentes en los huertos provienen sobre todo de la montaña. De estos intercambios surgen dos evidencias importantes a considerar. La primera es visible en el sentido de que la conectividad puede ser incentivada por el uso humano de las especies y la segunda gira en torno a cómo este uso puede generar las condiciones necesarias para que existan plantas arbóreas nativas, características del BMM. Destacamos, asimismo, que más allá de los convenios de protección, la presencia de especies importantes para el BMM en el huerto es promovida fundamentalmente por la intención y la voluntad de los pobladores representada por los diferentes usos en el contexto de la comunidad.

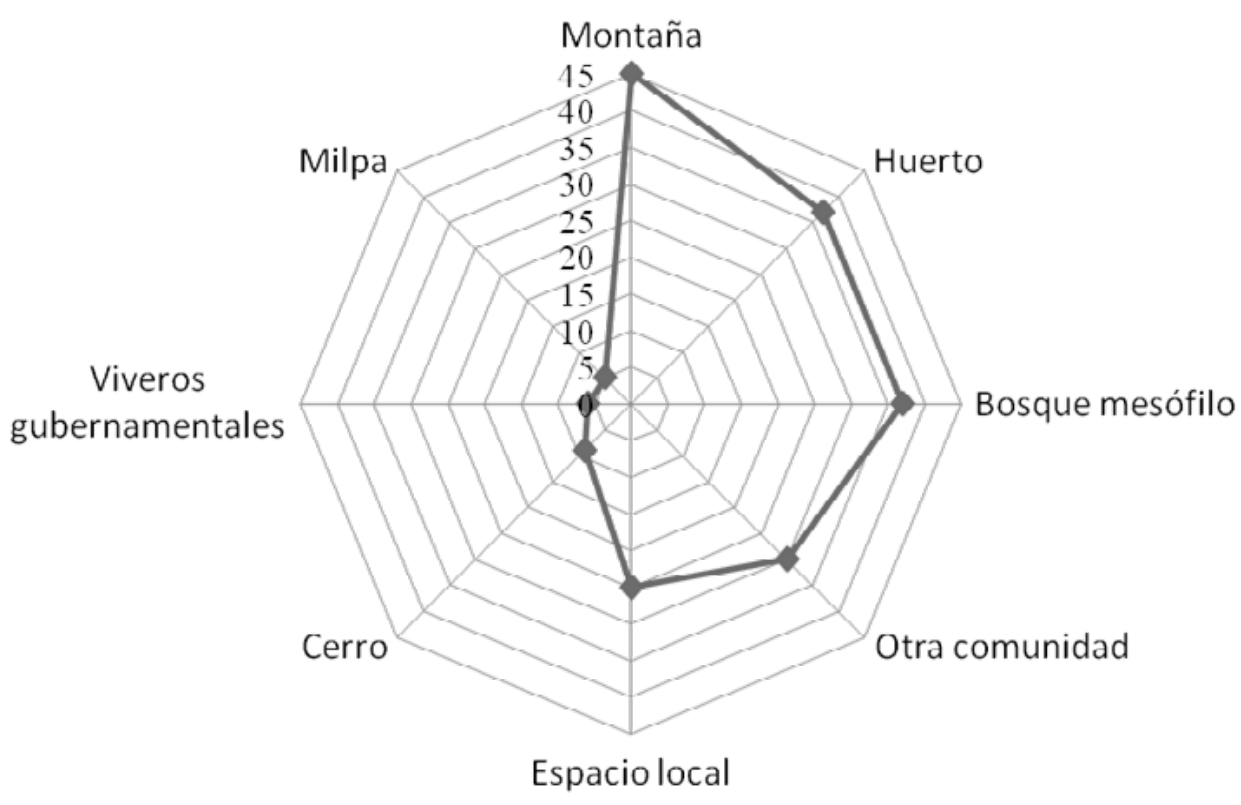

Figura 9. Número de especies encontradas por espacio de uso en la comunidad de Chiquihuite, Unión Juárez, Chiapas. 
Cuadro 1. Frecuencia (interacciones) con la que se encuentra representada

la especie entre todos los huertos muestreados.

\begin{tabular}{l|l|c} 
Especie & Nombre común & $\begin{array}{c}\text { Interacciones/total de } \\
\text { interacciones posibles }\end{array}$ \\
\hline $\begin{array}{l}\text { Agapanthus africanus (L.) Hoffmanns } \\
\text { Alnus acuminata Kunth }\end{array}$ & Agapando & $20 / 24$ \\
\hline $\begin{array}{l}\text { Roldana sp. } \\
\text { Zantedeschia aethiopica (L.) Spreng }\end{array}$ & Brotón & $20 / 24$ \\
\hline $\begin{array}{l}\text { Persea americana Mill } \\
\text { Prunus persica (L.) Batsch }\end{array}$ & Alcatraz & $16 / 24$ \\
\hline $\begin{array}{l}\text { Artemisia mexicana Willd. ex Spreng } \\
\text { Canna indica L. }\end{array}$ & Durazno & $14 / 24$ \\
\hline Sechium edule (Jacq.) Sw. & Estafiates & $13 / 24$ \\
\hline Baccharis vaccinioides Kunth & Mijahué & $12 / 24$ \\
\hline Saurauia aspera Turcz & Chayote & $11 / 24$ \\
\hline Tillandsia guatemalensis L. B. Sm. & Escoba & $11 / 24$ \\
\hline
\end{tabular}

\section{EL USO COMO APORTE A LA CONECTIVIDAD DE ESPACIOS}

En esta investigación hemos enfatizado la conectividad entre espacios de uso que, como hemos visto, es promovida por quienes propician los intercambios entre espacios productivos (como el huerto) y otros en aparente estado de conservación. No se trata de una acción aislada. Es una tarea relacionada con el uso que se da a los recursos vegetales y que permite a la población evitar gastos (Achá et al. 2007). Algunos estudios asumen como acción común el trasiego de plantas entre hogares o personas. Las mujeres zapotecas de los valles centrales de Oaxaca, por ejemplo, intercambian especímenes de los ecosistemas circundantes hacia los huertos por el gusto que les produce poseer plantas que sus vecinas no tienen, o por la valoración medicinal, ritual u ornamental que se les puede dar (Vásquez y Lope 2012). En la ciudad de San Cristóbal de Las Casas, Chiapas, 
grupos de personas locales y extranjeras han creado redes de este tipo que han ayudado a la conservación de especies y la reproducción de conocimientos para el mantenimiento de los huertos (Flores 2012).

En Chiquihuite, el principal incentivo para promover la conectividad entre los espacios de uso y el huerto es que las personas mantienen un modelo local de naturaleza basado en el siguiente principio: conservar es cuidar, cuidar de un terreno que sirve (A.A.P, entrevista personal, 3 de mayo de 2012). En este sentido, el huerto es un sitio que permite ahorrar recursos económicos, insumos y esfuerzo: es un lugar que sirve para ahorrar dinero - porque se obtienen de él algunos productos de autoabasto y se cultivan flores, su principal actividad económica-, tierra — se usa menor cantidad de área para sembrar maíz, pues el dinero que se obtiene de la venta de flores se utiliza para comprar este producto cuando el precio es menor-y trabajo —es el espacio que menos insumos y horas de trabajo necesita - ... si el jardín no estuviera, todos los demás - espacios- tampoco (F. V. L, entrevista personal, 21 de marzo de 2012).

Bajo esta lógica y siendo un lugar cercano a la casa, es en este sitio donde las personas prefieren «cuidar» las especies de la montaña a las cuales han otorgado un uso.

\section{EL PAPEL DE LAS ESPECIES VEGETALES, SU RELACIÓN CON EL USO, LA CONECTIVIDAD Y «EL CUIDAR»}

Las especies vegetales registradas interaccionan con distintos papeles dentro del huerto: son mayoritariamente nativas, arbóreas, tienen un uso diario para la comunidad y poseen algún estatus para la conservación. A partir de estas categorías se coteja esta información con las formas de uso (cuadro 2) y se observa que existe un porcentaje alto de especies comestibles, así como de uso ambiental, material y medicinal.

Investigaciones realizadas indican que de manera general es posible encontrar de 85 a 125 especies de plantas útiles en espacios pequeños alrededor de las casas (Alcorn 1994). Las que fueron halladas en el presente estudio están dentro del promedio de número de especies que se pueden encontrar en los huertos en México.

En los huertos de Chiquihuite, 36.70\% de las especies son nativas, 34\% neotropicales y $24.70 \%$ introducidas. En una investigación realizada en Alagoinha, 
Cuadro 2. Porcentaje de los principales usos de las plantas categorizadas en un estatus de conservación.

\begin{tabular}{l|c|c|c|c|c|c|}
\hline \% de uso & Total & Comestible & Medicinal & $\begin{array}{c}\text { Uso } \\
\text { ambiental }\end{array}$ & $\begin{array}{c}\text { Uso } \\
\text { social }\end{array}$ & Materiales \\
\hline NOM- ECOL-2010 & 8.3 & 2.75 & - & - & - & 2.75 \\
\hline CITES & 10.1 & - & - & 9.17 & - & - \\
\hline Lista Roja- UICN & 11 & 4.58 & 4.58 & - & - & 3.66
\end{tabular}

Brasil, se muestreó un total de 31 huertos y se encontró que hay una proporción igual (50\%) de especies nativas y no nativas (Albuquerque et al. 2005). Estudios en la región socioeconómica del Soconusco, Chiapas, donde se ubica nuestra comunidad de estudio, se determinó que en una muestra de 53 huertos de tres comunidades distintas, la riqueza de especies es de 490 y de estas $44 \%$ son nativas (Quique 2012). En las investigaciones sobre huertos se ha considerado que es común encontrar un porcentaje alto de especies introducidas debido a las plantas ornamentales (Niinemets y Peñuelas 2007). En los huertos muestreados, las especies nativas y neotropicales son las más representativas en número y tan solo $10 \%$ de especies ornamentales son introducidas.

Asimismo, los porcentajes más altos de las formas biológicas corresponden a las especies arbóreas (22\%) y arbustivas (15.55\%). En los huertos, dichas especies generan condiciones microclimáticas de humedad y luz, que permiten cultivar especies de pequeño porte (Lerner 2008). Es posible que en los huertos de la comunidad de Chiquihuite la presencia del estrato arbóreo y arbustivo promueva la presencia de especies herbáceas (37.60\%) y epífitas (17.43\%). De la misma manera, es probable que favorezca el número de especies características de BMM (se han encontrado 37), pues el componente arbóreo ha sido considerado un gran aliado para las especies típicas de este tipo de bosques, ya que este estrato les provee de las necesidades específicas para establecerse o les abastece de las condiciones para que puedan hacerlo en un mayor tiempo (Ramírez-Marcial et al. 2010).

Con respecto al uso, resalta que las tres categorías con mayor porcentaje son: el uso ambiental, con el $36.75 \%$; de esta proporción, $6 \%$ de las especies son usa- 
das para realizar prácticas de manejo en el huerto. Se citan ejemplos con algunas especies:

Pinus oocarpa y Pinus sp 1. Evita la pérdida del suelo por el exceso de lluvia. Es cultivada dentro de la comunidad, en espacios reconocidos como montaña, colindantes con aquellos considerados como milpa y huerto. La intención que antecede a esto es: «¿ves aquella montaña que se ve allá?, yo solito la sembré, son casi cinco hectáreas. No porque me dijeran, sino porque yo pienso. Mi casa está abajo. El pino tiene forma de triángulo y cuando le cae lluvia, lo para. Se Ileva menos la tierra y no se me viene pa' abajo. También me sirve pa' mis leñas» (E. D. L, entrevista personal, 10 de marzo de 2012).

Chiranthodendron pentadactylon. Aporta materia orgánica. Es de montaña. Una especie que se siembra especialmente en los huertos donde se cultivan flores de ornato. Esto es así por el aprecio que se tiene a la especie por ser «un árbol muy agradecido. Este nos sirve para abono. Nos sirve pa' que el alcatraz dé flor. Si no se pone este, no da» (G.H. L, entrevista personal, 2 de marzo de 2012). Cyathea fulva. Ofrece protección de los vientos para las plantas más pequeñas. Es un helecho arborescente que ha mantenido su presencia al «dejarse crecer solo» y de manera dispersa en un terreno. La planta, por su altura, funciona a manera de barrera para disipar los vientos «este 'chipé' lo deja pasar el ventarrón. En diciembre, qué ventarrones que vienen y lo despedazan toda la flor. ¡Eh, que lo lleva! Lo pongo así enfrente pa' que no» (L. H. L, entrevista personal, 18 de enero de 2012).

Miconia fulvostellata. Proporciona alimento a las aves que de otro modo consumen algunos de los productos que allí se cultivan. Es comúnmente llamada "cinco negritos», traída de la montaña y cultivada dentro de los huertos para evitar que los pájaros se coman los frutos que ahí se producen: durazno, ciruela y aguacate. La idea bajo esta práctica es que «los animalitos comen como nosotros, ellos también tienen derecho» (G.L.M, entrevista personal, 04 de abril de 2012). Con ello se impide que estas aves consuman los frutos que sirven a la población.

El resto de las especies de uso ambiental son plantas ornamentales. La principal actividad económica en la comunidad es la floricultura. Es posible que esta característica influya en la dominancia de este uso. La segunda categoría con mayor proporción de especies es la comestible, con $35.05 \%$, y en tercer lugar las que tienen uso medicinal, con $13.68 \%$. Estas características son muy similares a otros huertos. Por ejemplo, en Cuicatlán, México, en 30 huertos muestreados 
con 248 especies de plantas, $65.7 \%$ son ornamentales, $29.6 \%$ son comestibles y el $8.6 \%$ son medicinales (Blanckaert et al. 2004).

En relación con el estatus de conservación, a nivel de especie, observamos una relación directa entre diversidad de formas de uso y conectividad. Determinamos que a mayor número de formas de uso de las plantas se promueve un mayor número de intercambios entre espacios. Como ejemplo podemos citar el caso de Alnus acuminata. Es una de las especies arbóreas con mayor grado de conectividad entre los huertos y es característica de los BMM. Está protegida por la Lista Roja de UICN. En la comunidad es utilizada como combustible, como materia prima, y también tiene uso ambiental. Su cultivo es una práctica constante.

Por otro lado, podemos mencionar el caso de Cedrela odorata (cedro). También está presente en los BMM, restringida al rango de altitud más bajo. Está categorizada en el Apéndice III del CITES y es considerada especie vulnerable en la Lista Roja de UICN y sujeta a protección especial por la NOM-ECOL-2010. Sin embargo, la conectividad que presenta entre huertos es la más baja. Su uso es restringido a materia prima, específicamente en la elaboración de muebles para la casa. Se registró que su presencia en los huertos de Chiquihuite se debe a la promoción de su cultivo por programas gubernamentales.

\section{REFLEXIONES FINALES}

La conectividad de las especies vegetales en los huertos de Chiquihuite está íntimamente relacionada con el uso que se les da. Las más representadas son aquellas que tienen usos variados o representativos y no aquellas que están protegidas por uno o varios estatus de conservación. Los criterios para establecer estos convenios están generalmente basados en estudios de distribución y estado de poblaciones silvestres, principios bajo los cuales se ha asumido el concepto de conservación y sobre los cuales se ha edificado la importancia de una especie sobre otra. Para la comunidad, la presencia de especies importantes para los bosques se determina por medio del intercambio. A través de él se asegura y fomenta la presencia de especies que son útiles más allá del sentido biológico de la conservación. Por estas razones, la definición local de conservación no se puede explicar sin el uso. Este hecho constituye el principal hallazgo de esta investigación, pues nos aproximamos a una forma diferente de apreciar y enunciar el concepto de conservación. 
Entre los estudios realizados en otras latitudes y bajo otros contextos que han demostrado este hecho, cabe mencionar el análisis de Fraga (2006) respecto de una comunidad dedicada a la pesca, de la Reserva de la Biosfera Ría Lagartos, donde la conservación se estableció como una acción para establecer áreas reservadas para los «malos tiempos» y lidiar con la disminución en la captura de peces. El uso comestible local que se daba a los peces en la comunidad aseguraba la disponibilidad del recurso y la población de peces mantenía el número de individuos para satisfacer a la población. Berkes y Turner (2005) demuestran que el sentido de conservación entre los indígenas zapotecos de Oaxaca puede estar influenciada por el respeto hacia los recursos naturales y la prohibición de desperdiciar. Peres y Zimmerman (2001) determinaron que en el bosque tropical del Amazonas hay espacios cultivados y es posible encontrar ahí una gran cantidad de especies, tales como Swietenia macrophylla. Los nativos retienen en estos espacios especies que de otra manera permanecerían sin protección, debido a las altas tasas de deforestación en la zona. Bajo este sentido, se consideró a los nativos amazónicos como fuentes protectoras, creadoras de biodiversidad y promotoras de la conservación. El uso que le dan al recurso es para ellos el incentivo de su conservación.

Para una ANP como a la que corresponde la comunidad de Chiquihuite, la conservación es vista en términos de protección y aislamiento de la naturaleza. Este hecho niega la posibilidad de un balance entre producción y conservación (Toledo 2005). Para Chiquihuite hemos demostrado que el huerto funciona como un espacio donde, por medio del cuidado, se llevan a cabo estrategias de uso de la naturaleza, se mantienen interacciones entre unidades productivas y áreas en aparente estado de conservación, por lo que para establecer un verdadero manejo de los ecosistemas es importante que se considere «el cuidar» como otra forma de conservar.

\section{AGRADECIMIENTOS}

Al doctor Carlos Rommel Beutelspacher, profesor investigador de la Universidad de Ciencias y Artes de Chiapas, al M. en C. Óscar Farrera y al biólogo Francisco Najarro, del Herbario CHIP del Instituto de Historia Natural y Ecología del Gobierno del Estado de Chiapas, por su valioso apoyo en la identificación de las especies de este trabajo. 


\section{FUENTES DE CONSULTA}

Achá Cordero, Darío, Francisco Fontúrbel Rada, Diego Mondaca Gutiérrez, Iván Zambrana Flores, 2007, Introducción a la botánica: manual universitario, Bolivia, Publicaciones Integrales.

Albuquerque, Ulysses, Dárdano Andrade Lima y Javier Caballero Nieto, 2005, «Structure and floristics of homegardens in Northeastern Brazil», Journal of arid enviroments, 62, pp. 491-506.

Alcorn, Janis, 1994, «Noble savage or noble state?: northernmyths and southernrealities in biodiversity conservation», Etnoecológica, 3, pp. 7-19.

Barrera-Vásquez, Alfredo, 1981, "Sobre la unidad de habitación tradicional campesina y el manejo de recursos bióticos en el área maya yucatanense», Biotica, 5(3), pp. 115-128.

Berkes, Fikret y Nancy Turner, 2005, "Conocimiento, aprendizaje y la flexibilidad de los sistemas socioecológicos», Gaceta Ecológica, 77, pp. 5-17.

Blanckaert Isabelle, Ronny Swennen, Martín Paredes Flores, Rocío Rosas López, Rafael Lira Saade, 2004, "Floristic composition, plant uses and management practices in home gardens of San Rafael Coxcotlaán, Valle of Tehuacán-Cuicatlán, Mexico», Journal of Arid Environments, 57(2), pp. 179-201.

Breedlove, Dennis, 1981, Flora de Chiapas. Parte I: Introducción a la flora de Chiapas, San Francisco, California, Academia de Ciencias.

CITES, 2010, «Apéndices I, II y IIl», CITES, México, <http://www.cites.org/esp/app/appendices.php> [consulta: el 25 de marzo de 2013].

CONANP, 2008, «Programa de Conservación y Manejo: Reserva de la Biosfera Volcán Tacaná», SEMARNAT-CONANP, México, <http://www.conanp.gob.mx/que_hacemos/ pdf/programas_manejo/2014/VOLCAN_DE_TACANA_2014.pdf> [consulta: 03 de febrero de 2013].

Cook, Frances, 1995, Economic Botany Data Collection Standard, Inglaterra, Royal Botanic Gardens.

Cruz Hernández, Delmy Tania, 2010, «Apropiación territorial, conflicto e interfaz social: el caso de la comunidad de Chiquihuite en la Reserva de Biosfera del Volcán Tacaná, Chiapas», tesis de maestría inédita, San Cristóbal de Las Casas, Universidad Autónoma de Chapingo.

Durand Smith, Leticia, 2003, «¿Para qué sirven las Áreas Naturales Protegidas?», Ecología Política, 25, pp. 103-110.

Ellen, Roy y Simon Platten, 2011, "The social life of seeds: the role of networks of relationships in the dispersal and cultural selection of plant germplasm», Journal of the Royal Antrophological Institute, 17, pp. 563-584.

Escobar Hernández, María Eugenia, 2009, "Conocimientos y prácticas tradicionales vinculadas al desarrollo local sustentable de los mames en la Reserva de Biosfera Volcán Tacaná, Chiapas, México», tesis de licenciatura inédita, Tuxtla Gutiérrez, UNICACH.

Flores Reyes, Lorena, 2012, «Intercambio de plantas, semillas y conocimientos del huerto entre grupos culturales urbanos de San Cristóbal de Las Casas, Chiapas», tesis de maestría inédita, San Cristóbal de Las Casas, ECOSUR. 
Fraga, Julia, 2006, «Local perspectives in conservation politics: The case of the Lagartos Biosphere Reserve, Yucatán, Mexico», Landscape and Urban Planning, 74, pp. 285-295.

Galluzi, Gea, Pablo Eyzaguirre, Valeria Negri, 2010, «Homegardens: neglected hot spots of agro-biodiversity and cultural diversity», Biodiversity and conservation, 19(13), pp. 3635-3654.

Ghazoul, Jaoury y Douglas Sheil, 2010, Tropical Rain Forest Ecology, Diversity, and Conservation, Oxford, Universidad de Oxford.

González-Espinosa, Mario, Jorge Meave, Francisco Lorea-Hernández, Guillermo Ibarra Manríquez, Adrián Newton, (eds.), 2011, The red list of mexican cloud forest trees. Flora y Fauna, Fauna \& Flora International, Cambridge, UK.

Halffter, Gonzalo, 1992, "Áreas Naturales Protegidas de México: Una Perspectiva», en José Sarukán y Rodolfo Dirzo (comps.), México ante los retos de la biodiversidad, México, CONABIO, pp. 269-281.

Hammersley, Martyn y Paul Atkinson, 1994, Etnografía, Paídos Básica, España.

Ibarra-Manríquez, Guillermo, 1996, «Biogeografía de los árboles nativos de la Península de Yucatán: un enfoque para evaluar su grado de conservación», tesis de doctorado inédita, México, UNAM.

IMSS, 2012, Diagnostico anual de la comunidad de Chiquihuite, México, Unidad Médica Rural de Talquián.

Lazos-Chavero, Elena y Luisa Paré, 2000, Miradas indígenas sobre una naturaleza entristecida: percepciones del deterioro ambiental entre nahuas del sur de Veracruz, México, UNAM/Plaza y Valdés Editores.

Lerner Martínez, Tina, 2008, «lmportancia del huerto familiar Ch'ol en la economía campesina, el caso de Suclumpá, Chiapas, México», tesis de maestría inédita, San Cristóbal de Las Casas, ECOSUR.

Microsoft Office [en línea], 2010, [México]: Microsoft CLUF, 1990-2016. Programa informático.

Niinemets, Ülo y Josep Peñuelas, 2007, «Gardening and urban landscaping: significant players in global change», Trends in Plant Science, 13(2), pp. 60-65.

Paz, Fernanda, 2005, La participación en el manejo de áreas naturales protegidas: actores e intereses en conflicto en el Corredor Biológico Chichinautzin, Morelos, México, UNAM.

Peres, Carlos y Barbara Zimmerman, 2001, «Perils in parks or parks in perils? Reconciling conservation in amazonian reserves with and without use», Conservation Biology, 15(3), pp. 793- 797.

Proulx, Stephen, Daniel Promislow, Patrick Philips, 2005, «Network thinking in ecology and evolution», Trends in ecology and evolution, 20(6), pp. 345-353.

Quique, Rozen, 2012, "Cocoa, coffee and sugarcane farmers: an ethnobotanical characterization of their homegardens in the Soconusco región (Chiapas, México)», tesis de doctorado inédita, Montpellier, Laguedoc.

R Development Core Team, 2014, R: A language and environment for statistical computing, Austrria, R Foundation for Statitstical Computing.

Raffestin, Claude, 1980, Pour une geographie du pouvoir, Francia, Librerias Thecniques. 
Ramírez-Marcial Nepalí, Angélica Camacho-Cruz, Miguel Martínez-Icó, Alonso Luna-Gómez, Duncan Golicher y Mario González-Espinosa, 2010, Árboles y arbustos de los bosques de montaña en Chiapas, México, ECOSUR.

Ruiz Olabuenaga, José Ignacio y María Antonia Izpizúa, 1989, La descodificación de la vida cotidiana, España, Universidad de Deusto.

Rzedowski, Jerzy, 1983, Vegetación de México, México, Limusa.

SEMARNAT, 2010, Norma Oficial Mexicana NOM-059-ECOL-2010, Protección ambiental-Especies nativas de México de flora y fauna silvestres-Categorías de riesgo y especificaciones para su inclusión, exclusión o cambio-Lista de especies en riesgo, México, Diario Oficial de la Federación.

Shannon Paul, Andrew Markiel, Owen Ozier, Nitin S. Baliga, Jonathan T. Wang, Daniel Ramage, Nada Amin, Benno Schwikowski y Tey Ideker, 2003, «Cytoscape: a software environment for integrated models of biomolecular interaction networks», Genome Research, 13(11), pp. 2498-504.

Soulé, Michael, 1985, «What is conservation biology?», American Journal of BioScience, 35(11), pp. 727-734.

Taylor, Steve y Robert Bogdan, 1996, Introducción a los métodos cualitativos de investigación: la búsqueda de significados, México, Paidós.

Toledo, Victor, 2005, «Repensar la conservación: ¿áreas naturales protegidas o estrategia biorregional?», Gaceta Ecológica, 77, pp. 67-83.

Tropicos org., base de datos electrónica, Missouri Botanical Garden, <http://www.tropicos.org> [consulta: 2 de septiembre de 2015].

UICN, 2012, «Red list», <http://www.iucnredlist.org/> [consulta: 22 de octubre de 2012].

Vásquez-Dávila, Marco Antonio y Lope-Alzina Diana, 2012, «Manejo y Conservación de la Agrobiodiversidad y biodiversidad en huertos familiares de Oaxaca, México: un enfoque biocultural», en José, Flores-Guido (comp.), Los huertos familiares de Mesoamérica, Mérida, Universidad Autónoma de Yucatán/Conacyt, pp. 280-308.

WRI, 1992, "Biodiversity Glossary of terms», <http://www.igc.org/wri/biodiv/gbsglos. html> [consulta: 18 de octubre de 2012].

Fecha de recepción: 29 de noviembre de 2014

Fecha de aceptación: 6 de octubre de 2015 